\title{
1 \\ Importance and Use of Simulation in a Cyber Environment
}

\author{
M. Uretsky \\ Information Systems Department, Stern Graduate School of \\ Business, New York University, 44 West 4th Street, New York, \\ NY 10012, USA \\ muretsky@stern.nyu.edu
}

\begin{abstract}
The thesis is very simple: Simulation is the future! Growing environmental complexity leaves few alternatives to the use of simulation for planning, controlling and training. We can stipulate to the existence of a cyber environment. There are few who would question the fact that more and more of our activities are dependent upon both sophisticated, interconnected models and computers on which to executive them. Moreover, attendant with this growth are an increasing number of practical operating problems and risks. The thesis of this paper is that simulation offers one of the most fruitful ways to address these issues. Indeed, increasingly, simulation may be the only way to effectively address them. If one agrees with this conclusion, it follows that there is a research agenda that must be implemented to resolve critical open issues.
\end{abstract}

\section{Keywords}

simulation, training, computers, research 


\section{INTRODUCTION}

The thesis will be developed in the following manner. We begin by briefly reviewing critical dimensions of three related areas - the cyberspace environment and activities taking place within it, simulation and gaming, and human learning and performance. Using this survey as a basis, we will move on to show that growing system complexity carries with two major problems with it - people become increasingly removed from systems they use and problems will inevitably happen. Simulation appears to be one of the more fruitful tools for addressing these problems. At the same time, there are clear gaps that must be filled in before this potential can be realized.

\section{BACKGROUND - SELECTED PIECES}

Examining the role of simulation in addressing cyberspace problems requires that we agree upon the nature of the environment, the capabilities of simulation, and how these factors relate to our human behavior.

\subsection{The Cyberspace Environment}

The thing called cyberspace represents both little change from the past and simultaneously radical departures. As a practical matter, the computer-based environment has been present since the early ' $60 \mathrm{~s}$. We have been evolving towards larger and more complex computer-based systems that are increasingly interconnected. With the rapid growth of the world wide web, the changes have become so great and rapid that they have the potential for altering some of our most fundamental assumptions about our environment and the ways with which we interact with each other and this environment.

\section{Growing Automation and Dependence Upon It}

We live in a computer-based environment. Literally every aspect of our lives depends upon or is impacted by computers. Every device we use, from watches to televisions to cars to medical diagnostic equipment, depends in one way or another on computer chips that have been embedded within them. At the other end of the spectrum are large-scale, so-called mainframes, that are used for everything from meteorological forecasting to high volume transaction processing. In between are, of course, the ever present notebook and desk top computers we all use to carry out our daily business.

These computers are used for everything from word-processing, to spreadsheetbased analyses, to execution of highly complex, sophisticated mathematical algorithms. Unlike the past, most of the programs are bought from independent vendors, presumably because of their functionality. (I say presumably, because few of us actually run adequate functionality tests). The important factor is that, unlike past practice in which we wrote our own computer programs, we now rely upon software written by other people. Even where we develop our own modules, we are likely to embed code modules developed elsewhere. Stated differently, we are using computer code developed by other people and we are assuming that the 
people are competent, that they have adequately tested their product, and that it satisfies our required functionality.

And finally, the models and the computers are interconnected. Computer models pass data to other models and to users. In the same sense, computers communicate with each other over short or long distances - and without any significant human intervention.

\section{Network is the Focus}

As significant as any of the above features might appear, they have now lost their real significance. Most observers have shifted their attention away from the individual components mentioned above, to the relationship between them. Stated another way, the future is in the network, not the computer! When viewed from this perspective, The computer, is just one specialized component within the bigger picture.

There are two very separate views of this inter-connectivity. Consider the world wide web as one example. From the perspective of the user, inter-connectivity has only three components, i.e., the user, the browser, and content of the sites accessed through the various search engines.' All of this is independent of the fact that from the scientist's perspective, everything is dependent upon a complex, ever-changing infrastructure and network topology.

The concept of inter-connectivity is not new. EDI has been around for many years. The essential point is that, with the web, inter-connectivity has become so common, that nobody cares about the individual components.

It is essential to recognize that the automatic electronic transfer of data between different modules or locations has the effect of creating tightly a tightly-coupled system. In such a case, the output from one module are transferred to another, with little opportunity for human intervention or at least implicit quality control.

\section{Complexity and Ignorance}

The systems we are using have gotten larger and more complex. Each individual module is larger than it's predecessors. Increasingly, these modules utilize complex programs or concepts that are beyond the immediate knowledge of the professionals using them. And finally, the modules are inter-connected with others - at the same locations or elsewhere. The systems are increasingly big and complex. All of this means that users are less able to understand the tools that they are using - which may be acceptable if the tools work (and you know they are working satisfactorily), but certainly not in times of difficulty.

\section{Virtuality}

What you see is what you get. Since the network is the dominant factor, one is almost always dealing with entities that are geographically located someplace else. This focus shifts away from the physical and reality is thus largely a function of abstract or second-order perceptions. In this sense, an organization exists because it is on the network, has a site and called an organization - virtual firms, virtual

\footnotetext{
${ }^{1}$ The computer, per se, has been omitted, since it is functionally transparent. While we are using personal computers today, we may well be using telephone devices, cable television, or what is called in the US, WebTV.
} 
organizations, virtual shopping malls, etc. They have many of the characteristics of their counterparts, but they may or may not have real physical existence. As a practical matter, this means that one can be dealing with virtual employees, organizations, or firms. A location on the web can be more important than a real physical location. And finally, the fact that existence lies in a virtual reality introduces new concept in alien behavior. A person or organization can literally be doing business in a country, without being physically present, even though performing these same activities in person might be illegal. A visitor to a web site can be just as unwelcome as he or she might be in person.

\section{Fluidity and Fast response}

Constant change is the norm. The world wide web is constantly and speedily evolving. New organizations are forming and disappearing. The old idea of products with multiple year expected lives is rapidly being replaced. The ease of product creation and market entry creates competitive forces that shorten a product's expected life to well under a year. The speed with which things change puts a premium on fast response, delivery and adaptation. In some ways, management of information about economic activities can be just as (more) important than the activities themselves.

It is difficult to set long term directions in a period of fluidity and fast response. The financial markets (and the press) look for immediate indicators of good and bad performance.

\section{Trust issues are common denominators}

The environment described above carries with it a large number of unsolved and evolving issues. The resolution of these issues will form the basis for the coming age of electronic business.

A natural by-product of the previously noted phenomena is growing concern with trust-related issues. Without trust, there can be no real reliance - and growth. The trust issues come in many forms. While it is beyond the scope of this paper to deal with electronic commerce or trust per se, enumerating some of these areas does serve to highlight the breadth of concerns.

- Security. There is a constant stream of articles regarding security violations associated withweb use. While there are certainly important technical issues, the most crucial concern is the lack of trust in the systems being used. Credit card use on the Internet is one example. There are many articles about the possible theft and misuse of numbers. At the same time, from a realistic point of view, the incidence of these events is extremely rare and you are probably safer using a computer-based system than giving your credit card to someone over the phone or in a restaurant.

- Contracting. There are a broad range of what might be called contracting issues. The legal structure is just evolving. There is currently no electronic concept of a notary public. There is no agreement about the location of the transactions. (If you are in New York accessing a gambling casino in the Bahamas, where is the transaction taking place? Are you violating any laws? Do you know what laws are applicable?) 
- Intellectual property rights. Who owns your ideas and how can you protect them.Some countries, such as the United States, have very well-defined, restrictive concepts of ownership embedded within their patent and copyright laws. The rationale is associated with providing economic incentives to encourage innovation. Other countries, particularly developing nations; view free distribution of things like software as being more important than the protection of developers.

- Organizational change.The web is eliminating the need for middle level workers. (It may not be actually eliminating the workers, since union regulations act as a strong retarding force in some countries). This is a very important point and one that comes to the heart of our thesis. Eliminating middle level workers flattens organizations. It creates virtual organizations, with workers located in many different places - often not available when they might be needed. Thus, in a time of increasing complexity, we are increasingly working with distributed knowledge and workers who may not be available when they are needed.

\subsection{Simulations}

A simulation is a model. It is designed to represent the important characteristics of some system. Underlying this simple statement lie a number of important assumptions.

- A simulation is a representation of something. It is not the thing itself.

- To be useful, the simulation must represent the important characteristics of the thing being simulated. This means that a particular use is contemplated and that someone has made a value judgment regarding the features that are and are not important. This is a very important set of decisions, since they drive the tests needed to validate the models. A model being used for training purposes need only have sufficient face validity to cause the trainees to behave in the desired manner. In contrast, the model being used for forecasting or decisionmaking purposes must have all of its underlying assumptions tested (individually and together) and validated.

- From a conceptual point of view, the form of the simulation is irrelevant. It does not matter whether it is a role playing exercise or a more formal attempt to get at model-generated 'what-ifs.'

Traditionally, most simulations have existed within academic frameworks. This is changing, we are now seeing an increasing number of them used within business contexts. Subject to the above noted caveats, they can be used in industry when one or more of the following conditions are satisfied:

- It is essential to collapse time, permitting experimenters or participants to get immediate feedback on their potential decisions. 
- The only way to test a system is through simulation. Pilots and nuclear plant operators rely heavily on simulation-based training in critical events because other alternatives are either not feasible or ineffective.

- The cost of doing a simulation is less costly than the cost of dealing with reality.

\section{CYBERDOOM: RISK-RELATED ACCIDENTS}

The risks associated with modern systems are bound to remain. Moreover, as low as the related probabilities might be, we need to take any feasible steps to mitigate the expected damages.

Note that the above comments are premised on an assumption that the risks are unavoidable. This is consistent with the line of reasoning presented by Charles Perrow (1984). His line of reasoning was very straight-forward:

- All systems (and their component modules) can fail. There is no such thing as a fully debugged module. Indeed, within the computer environment, we are even limited in our ability to measure reliability in any meaningful sense.

- Systems grow and as they grow, they get more complex. Systems are rarely smaller and simpler than their predecessors. We build systems by adding features and combining previously separate modules. As the models get larger and connected, the number of possible combinations increases.

- Automation of systems makes them more tightly coupled. One of the features of automation is the linking of previously separate modules. This means that information from one module is automatically transmitted to the next automated module - thus eliminating opportunities for traditional, human, supervisory review, prior to use of the data by the next module. Stated another way, an error in one module will be automatically transmitted to the next. Unless someone recognized the error in advance and made provision for handling it, it will likely be used!

- As system grow and get more complex, they get less understandable. A large system is less understandable than a smaller one with fewer features. The lack of understandability is a function of two factors. First, the large number of components and component features strains the ability to keep track of everything and understand both the components and the entity as a whole. And second, the large number of people involved with the design, implementation and operation of these systems introduces organizational problems that also reduce the ability to understand what is taking place - and why.

- Early symptoms of system problems are either missed or discounted. Some early symptoms of problems are bound to be missed. Some may simply be missed because the operators are focusing elsewhere. Some may be below the thresholds causing us to focus on them. Some may be missed because the operators lack the experience to recognize them or evaluate their significance. 
And finally, others will be implicitly missed, because they are ambiguous and the operators take a 'wait and see' approach rather than take the consequences of taking what might be untimely corrective action.

- And finally, the ability of organizations to deal with these problems is limited by the their own structure and behavior. Given that the problems are recognized, organizational forces will cause corrective action to be, at best, delayed while damage continues. This phenomenon is found in many different contests - "It's not my job." "I'm looking for my supervisor."

The bottom line is quite clear, accidents will happen - probably with less frequency than in the past - and when they do, they will be serious. There is no shortage of examples of these phenomena - Three Mile Island, Bophal, Challenger, and on and on.

I am very concerned about this situation. I think that is more serious than might appear on the surface. We are producing managers who enter the managerial ranks without developing the experiences (intuition) gained as a by-product of working one's way up the ranks. We are using managerial work stations with imbedded technologies that are not, and cannot be, understood by their typical users. Intelligent agents and genetic algorithms a giving some web-based modules the ability to change and mutate without human intervention. And finally, we are producing managers who view the use of technology in the same way we all view using a car. (You turn the key and it works. Moreover, using it does not require understanding all of it's component parts).

BRINGING IT TOGETHER: DIRECTIONS FOR THE FUTURE

The thesis of this brief paper is that simulation should be looked at as an increasingly important tool for addressing the kinds of problems discussed above. While one could make a case for the fact that this is the most cost-effective approach, it is equally reasonable to say that it is the only real alternative. This observation is based on the fact that, much as some people might like, systems will continue to be increasingly large, complex, and automated. We will also never return to an environment in which people literally work themselves up the ladder from the loading docks. Intuition based on experiences having a physical reality are largely gone and they will not return.

The ability to use simulation to address these problems is present, but it has yet to be adequately developed. Three sets of capabilities must be present to make meaningful progress: (1) an ability to develop adequate and appropriate simulations, (2) an ability to run these simulations properly, and (3) an ability to adequately evaluate their results.

- Development. We have the ability to develop simulations. There is a respected literature on simulation and game development. While much of this literature is anecdotal and it is dispersed over different disciplines, it does exist and appears to be serving its audience adequately. 
At the same time, we have not ratcheted up our development capabilities adequately. Most computer-based simulations and games are still written in basic computer languages, like Visual Basic and Access. And we have failed to take advantage of the broad range of developmental efficiencies associated with object-oriented approaches. This development is essential, since it permits moving and embedding knowledge into new applications.

- Running. There is a body of professionals who have experience in operating simulations. These professionals are dispersed over different disciplines operations research, engineering, sociology, and business management. At the same time, it is equally useful to recognize that, in spite of the existence of clusters within lots of organizations, there is no generally accepted gathering place for professionals in this area!

- Evaluating. As shown below, there has been relatively little formal work associated with validation of the simulations and their results. Unfortunately, the quality of work done to date has been open to serious question - my opinion. This gets at the heart of the trust issues noted above. If we, as professionals, cannot provide adequately defensible validation data, we are relegated to be just another striving group of disciples - and like similar groups in the past, often unheard or ignored.

We need to address several critical issues if professions, like this, are to realize their critical roles in future economic developments.

- We must deal with the honest observations that, outside of engineering and academic sociology, there is not general acceptance of this approach.

- There are no agreed upon texts or guidelines or standards.

- This is not yet a recognized academic or business discipline or are of expertise. It should be, but it is not yet there.

In brief, we have a long way to go. I do not question the need for progress. Indeed, I hope I have made a case for simulation as an essential management and research tool in our rapidly changing web-based environment. At the same time, while some of the underlying methodology is present, there are a large number of issues that must be addressed adequately before real progress can be made.

\section{A RESEARCH AGENDA}

Assuming that one buys into my assertion that simulation is a critical tool for future management and training, there are a large number issues that must be addressed. The following research agenda has it's origin in a list provided in Greenblat and Duke (1975). They point out that a large number of assertions have been made in the literature regarding the potential benefits associated with simulation-gaming for training purposes. Their list is broken into the following major categories: 
- Participant motivation and interest

- Cognitive learning

- Impact on the character of later course work

- Affective learning regarding subject matter

- General affective learning

- Changes in classroom structure and relations

Each of these categories are subdivided into more pointed sets of topics within the original source. Elaborating upon them is beyond the scope of our immediate effort and you are directed to the Greenblat and Duke material.

The critical point regarding these potential benefits is that they are allegations or hypotheses awaiting further testing. Little respectable progress has been made since the '70s!

Going beyond the training and education environments requires addressing a broad range of far more serious questions.

- Model validity. Training and educational use requires only that the models have face validity. They must be adequately believable by their users. Adequacy, in this sense, is measured by the fact that people respond in an intended fashion. Any decision-making or predictive use of simulation requires that one be able to guarantee structural validity and predictive ability of both the model and data. This ability is easier in straightforward simulations that are mathematically oriented, than in complex simulations with large behavioral components.

- Impact - the final frontier. If simulation is to be a meaningful and relied upon tool in the cyber environment, we need to answer some very important and immediate questions.

* What effect will it's use have on organizational performance? Will people who use simulations be able to communication and operate within organizations more effectively than others? Will they have more empathy and understanding of those around them?

* Will people who have used simulations be able to generalize their knowledge into new and somewhat different situations?

* Will people who have used simulations be able to respond to emergencies - especially those requiring fast response time and dealing with complexity, areas calling for a high degree of intuitive behavior?

Simulation, on paper, is a very powerful and useful technique. It has the ability to help managers address some very critical issues. Indeed, it may be the only approach available in many cases. At the same time, practitioners of this field have a long way to go to validate the value of their tools. This paper has briefly outlined the environment and the need. The issues listed are just the tip of the iceberg. Add to them and address them. 


\section{REFERENCES}

Greenblat, Cathy S. (1975) Teaching with Simulation and Games: A Review of Claims and Evidence," in Greenblat, Cathy S. and Duke, Richard D., Simulation-Gaming: Rationale, Design, and Applications, New York: Halstead Press, pp. 273-274.

Perrow, Charles (1984) Normal Accidents: Living With High-Risk Technologies, New York: Basic Books

\section{BIOGRAPHY}

Mike Uretsky is Chairman of the Information Systems Department and Professor of Information Systems at New York University's Stern School of Business Graduate Division. In this position, he develops and runs seminars dealing with the impact of information-related technologies on company and industry performance. The current focus of these activities centers around the changes of widespread digitization on the broadly defined media industry. He is a leader in developing and implementing innovative training programs. He is a well-known developer and user of management training simulations - programs that simulate changing business conditions usingprocesses similar to those found in aircraft simulators. 